\title{
Compact Circular Polarization Design for Equilateral Triangular Micro Strip Antenna
}

\author{
Manidipa Roy, Ashok Mittal
}

\begin{abstract}
This article focuses on designing a single-feed circularly polarized equilateral triangular microstrip patch antenna. The axial ratio bandwidth of the antenna is around 190 $\mathrm{MHz}$. The antenna has been etched at specific locations for achieving circular polarization. The suppression of surface waves is also being focused upon for gain enhancement. The array of cylindrical metallic pins is embedded near the radiating side of the patch antenna. The gain enhancement of around 3.23 $d B$ is observed. The antenna is designed for use in satellite communications.
\end{abstract}

Index Terms: compact, circular polarization, slots, axial ratio.

\section{I.INTRODUCTION}

The planar, small-sized microstrip patch antennas embedded on thin dielectric substrates are extensively used for mobile and wireless applications. Alongwith other advantages the microstrip patch antennas find its place in modern communications because of its capability to radiate circularly polarized waves. The circular polarization can be obtained by using dual feed antennas[1-2]. The dual feed of 90 degrees phase and equal magnitude are being fed at the two specific locations on the metallic patch antenna surface. This produces two near degenerate orthogonal modes in the antenna. The single feed antennas[3-4] have specific surface perturbation so as to produce two orthogonal modes, with slightly different current distributions along two orthogonal axes.

This article focuses on the generation of circularly polarized radiation in an equilateral triangular antenna with slotted geometry. The slots are being etched to obtain compactness in the antenna design. The gain improvement is also being focused upon, for this the cylindrical metallic pins are being embedded near the radiating edge of the antenna to suppress the surface wave propagation.

\section{ANTENNA DESIGN}

The slotted geometry of the patch is also being improvised here in this article so as to obtain compact antenna characteristics from the same patch antenna. The perturbations are adopted for compactness of the patch antenna. The electrical length of the antenna is being

Revised Version Manuscript Received on July 20, 2019.

Manidipa Roy, Department of USICT, GGSIPU, Delhi

Ashok Mittal, Department of Electronics and Communication Engineering, AIACT\&R, Delhi modified. The antenna designed is an equilateral triangular patch antenna operating at $13 \mathrm{GHz}$. The objective behind using this patch is to achieve compact design and two achieve circular polarization by generating two near degenerate orthogonal modes. The advantages of using this patch antenna is that the radiation characteristics are nearly same as that of rectangular patch antenna and the design is compact. The size is further reduced by introduction of slots in the patch antenna surface. The cylindrical metallic pins are introduced for enhancing the gain characteristics.

The triangular patch antenna is designed. The resonant frequency of the equilateral triangular patch is [1]

$$
f_{Y}=\frac{c k_{m n}}{2 \pi \sqrt{\varepsilon_{y}}}=\frac{2 c}{3 a \sqrt{\varepsilon_{y}}} \sqrt{m^{2}+m n+n^{2}}
$$

The dimension of the patch antenna follows the given radiation condition [1-4]

$$
\frac{\Delta S}{S}=1 / 2 Q_{0}
$$

where $\Delta \mathrm{S}$ is the area perturbed in the patch antenna $\Delta S=\Delta S_{1}+\Delta S_{2}$

$\Delta S_{1}$ and $\Delta S_{2}$ are the areas of the slotted sections perturbed

$\mathrm{S}$ is the area of the patch

$Q_{0}$ is the quality factor of the patch antenna.

The circularly polarized rectangular patch antenna is designed at $13 \mathrm{GHz}$ on FR4 Epoxy substrate. The quality factor[4] is

$$
\frac{1}{Q}=\frac{1}{Q_{\text {rad }}}+\frac{1}{Q_{\text {sw }}}+\frac{1}{Q_{d i}}+\frac{1}{Q_{c u}}
$$

The term involving $Q_{\text {SIV }}$ is associated with the surface wave loss, $Q_{d i}$ is associated with dielectric loss and $Q_{c u}$ is associated with Copper loss.

$$
Q_{d i}=1 / \tan \delta ; Q_{c u}={ }^{t} / d_{g}
$$

here $d_{g}=(\pi f \mu \sigma)^{-1 / 2}$ is skin depth of the conductor.

The $Q_{\text {rad }}$ is given by

$$
Q_{\text {rad }}={ }^{2 \omega \varepsilon_{h} K} / t\left(\frac{G}{l}\right)
$$

here $\left(\frac{G}{D}\right)$ is associated with conductance per unit length of radiating cavity and

$$
\text { here } K=\iint|E r|^{2} d A /\left(\int_{0}^{\text {permeter }}|E r|^{2} d l\right)
$$

In a rectangular/square patch operating in the $T M_{10}$ mode, $K$ is $b / 4$ and $\left(\frac{G}{i}\right)$ is $\frac{G_{\text {rad }}}{a} \times$ Here

$$
G_{\text {rad }}=\frac{1}{R_{\text {rad }}}
$$

here $R_{\text {rad }}$ is the radiation resistance and here $\mathrm{G}_{\mathrm{rad}}$ is the radiation conductance.

$$
R_{\text {rad }}=\frac{Q_{r}}{\omega C_{10}}
$$

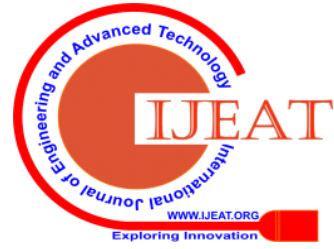




\section{Compact Circular Polarization Design for Equilateral Triangular Micro strip Antenna}

here $Q_{r}$ is the radiation quality factor $Q_{r}=\operatorname{Re}\left(k_{10}\right) / 2 \operatorname{Im}\left(k_{10}\right)$

where,

$k_{10}=\left(\frac{\pi}{b}\right)-\left(\frac{\Delta_{4}}{b}\right)$

here

$\Delta_{p+1}=\frac{2\left(a_{10} b\right)\left(\pi-\Delta_{p}\right)}{\left(\left(a_{10} b\right)^{2}+2 \Delta_{p} \pi-\Delta_{p}{ }^{2}-\pi^{2}\right)}-\frac{\Delta_{p}{ }^{a}}{3}$

with $\Delta_{0}=0$ as seed value.

$\alpha_{10}=j 2 \pi Z_{0} Y_{w} t / \lambda_{0} a$

here $a, b$ are radiating (width) and non- radiating (length) edges of the rectangular patch antenna respectively.

where $t$ is thickness of the substrate,

$Z_{0}$ is characteristic impedance of the patch,

$\lambda_{0}$ is wavelength at the operating frequency,

$Y_{w}$ is wall admittance of the patch cavity,

and $Y_{w}=G_{w}+j B_{w}$

where $G_{W}$ is wall conductance

and $B_{w}$ is wall susceptance

These values are mathematically calculated using relations

$$
\begin{aligned}
& G_{W}=\left(\frac{\pi}{376}\right)\left(\frac{a}{\lambda_{0}}\right) \\
& B_{W}=0.01668\left(\frac{\Delta_{1}}{t}\right)\left(\frac{a}{\lambda_{0}}\right) \varepsilon_{e}
\end{aligned}
$$

where

$$
\left.\frac{\Delta_{1}}{t}=0.412\left(\left(\varepsilon_{e}+0.300\right) /\left(\varepsilon_{e}-0.258\right)\right)\left(\frac{a}{t}+0.262\right) /\left(\frac{a}{t}+0.813\right)\right)
$$

and $\varepsilon_{e}$ is an effective dielectric constant

$$
\varepsilon_{\theta}=\frac{\left(\varepsilon_{h}+1\right)}{2}+\left(\frac{\left(\varepsilon_{h}-1\right)}{2}\left(1+\frac{10 t}{2}\right)\right)^{-1 / 2}
$$$$
\text { and } C_{10}=(1 / 2) C_{d c} \cos ^{-2}(\pi / 0 / b)
$$

$\left(x_{0}, y_{0}\right)$ are the co-ordinates of the feed point for co-axial feed

$C_{d c}$ is dc patch capacitance $(\varepsilon a b / t)$

A circularly polarized equilateral triangular patch antenna is designed which satisfies the above mentioned equations. The antenna has been slotted at three positions for achieving circularly polarized radiation and an array of shorting pins has been embedded in the dielectric substrate of the radiating patch edge. The improvement in gain is observed after embedding cylindrical metallic pins in the dielectric substrate.

The parametric study of the dimensions of the slots was carried out. The side length of the square patch is $14 \mathrm{~mm}$ and the perturbed triangular areas are having $1.8 \mathrm{~mm}^{2}$ and 1.85 $\mathrm{mm}^{2}$ respectively. The dimensions of all the slots are same with a length of $12 \mathrm{~mm}$ and width of $1 \mathrm{~mm}$. The resonant frequency comes out to be around $8.9 \mathrm{GHz}$ after etching the slots on the patch surface. The axial ratio bandwidth is around $100 \mathrm{MHz}$ for the modified patch antenna.

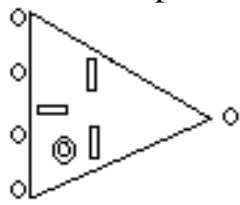

Fig. 2. Proposed circularly polarized Antenna design

\section{Parametric Analysis}

Parametric Study of dimensions of the slots has been done. The length and width of all the slots are same. The table below shows the parametric study of the slots done in the proposed structure.

Table1. Parametric study of the slot width

\begin{tabular}{|c|c|c|}
\hline S.No. & $\begin{array}{l}\text { Slot } \\
\text { length }(\mathbf{m m})\end{array}$ & Gain(dB) \\
\hline 1 & $0.148 \lambda_{0}$ & 1.71 \\
\hline 2 & $0.207 \lambda_{0}$ & 2.44 \\
\hline 3 & $0.267 \lambda_{0}$ & 3.12 \\
\hline 4 & $0.326 \lambda_{0}$ & 5.67 \\
\hline 5 & $0.356 \lambda_{0}$ & 6.98 \\
\hline 6 & $0.415 \lambda_{0}$ & 4.70 \\
\hline
\end{tabular}

Table2. Parametric study of slot length

\begin{tabular}{|c|c|c|}
\hline S.No. & $\begin{array}{l}\text { Slot } \\
\text { length(mm) }\end{array}$ & Gain(dB) \\
\hline 1 & $0.148 \lambda_{0}$ & 1.71 \\
\hline 2 & $0.207 \lambda_{0}$ & 2.44 \\
\hline 3 & $0.267 \lambda_{0}$ & 3.12 \\
\hline 4 & $0.326 \lambda_{0}$ & 5.67 \\
\hline 5 & $0.356 \lambda_{0}$ & 6.98 \\
\hline 6 & $0.415 \lambda_{0}$ & 4.70 \\
\hline
\end{tabular}

The improvement of $3.23 \mathrm{~dB}$ is observed after embedding shorting pins.

\section{RESUlts}

The novel structure has been simulated on Ansoft HFSS v12 software and fabricated. The simulation and fabrication results are shown:

Fig. 3 Circularly Polarized Antenna structure fabricated

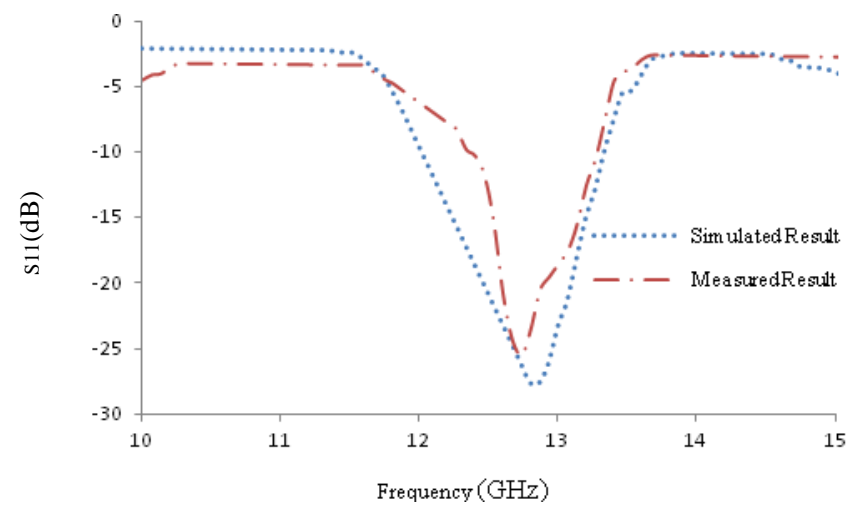

Graph1. Graph showing the reflection coefficient characteristics for the novel circularly polarized antenna 


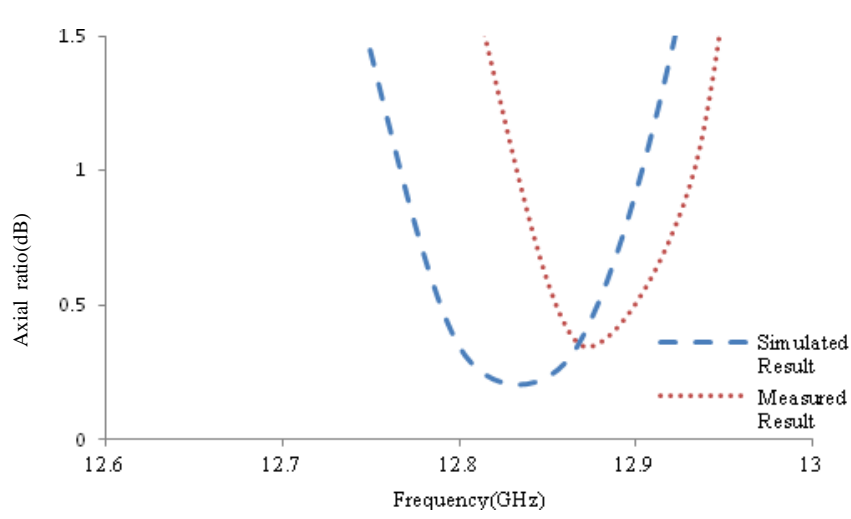

Graph2. Graph showing the axial ratio characteristics for the novel circularly polarized antenna

\section{Conclusion}

The circularly polarized antenna has been designed theoretically. Apart from obtaining circular polarization the compactness of the antenna is also being focussed upon the antenna is designed at $12.8 \mathrm{GHz}$ initially to produce the circularly polarized radiation. The modified antenna is suitable for its use in satellite applications.

\section{REFERENCES}

1. Ramesh Garg, Prakash Bhartia, Microstrip Antenna Design Handbook, Artech House, London

2. J.R.James, P.S.Hall, Handbook of Microstrip Antenna, Peter Peregrinus, London

3. C. A. Balanis, Modern Antenna Handook, Wiley \& Sons, Hoboken, NJ, 2008

4. Keith R. Carver, James W. Mink, Microstrip Antenna technology, IEEE Transactions on Antennas and Propagation, Vol.Ap-29,No.1, January 1981

5. G. Steven, Q.Luo and F. Zhu, Circularly Polarized Antennas, John Wiley \& Sons, UK, 2014.

6. T. Cai, G. Wang, X. Zhang and J. Shi, Low-profile compact circularly polarized antenna based on fractal metasurface and fractal resonator, IEEE Antennas Wireless Propagation Letters, Vol. 14, pp. 1072-1076, 2015

7. Nasimuddin, X. Qing and Z. N. Chen, Compact circularly polarized slotted patch antenna for GNSS applications, IEEE Trans. Antennas Propagat., Vol. 62, No. 12, pp. 6506-6509, 2014.

8. V.V. Reddy and N.V.S.N. Sarma, Compact circularly polarized asymmetrical fractal boundary microstrip antenna for wireless applications, IEEE Antennas Wireless Propag. Lett., vol. 13, pp. 118-121, 2014

9. W.S. Chen, C.K. Wu, K.L. Wong, Inset microstripline-fed circularly polarized microstrip antennas, IEEE Trans. Antennas Propag., vol. 48, no. 8 , pp. $1253-1254,2000$

10. Rajkishor Kumar and Raghvendra Kumar Chaudhary, Circularly polarized rectangular DRA coupled through orthogonal slot excited with microstrip circular ring feeding structure for Wi-MAX applications, International Journal of RF and Microwave Computer-Aided Engineering, Volume 28, Issue 1, January 2018

11. Manidipa Roy, Prateek Juyal, "Surface Wave Reduction in Circularly Polarized Microstrip Patch Antenna mounted on Textured Pin Substrate", IEEE AEMC 2011

\section{Authors Profile}

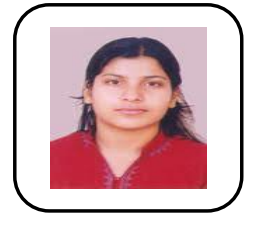

Manidipa Roy is M.Tech. in RF and Microwave Engineering. She had been associated with AIACT\&R, Delhi. Currently she is working as Assistant Professor in ABES Engineering College, Ghaziabad, Uttar Pradesh. She has teaching and research experience of around seven years. She had been a Research fellow at Ambedkar Institute of Advanced Communication technologies and Research, Delhi. She had guided many under graduate and post graduate students in their projects. She has several publications in International and National Journals and Conferences. She is involved in several Sponsored projects and consultancy projects. She has been awarded Gold Medal in M.Tech. RF and Microwave from Guru Gobind Singh Inderprastha University, Delhi.

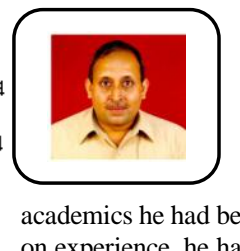

Dr. Ashok Mittal is M.Tech. in Microwave Electronics from University of Delhi, Ph.D. from Faculty of Technology, Delhi College of Engineering, University of Delhi. He has Teaching and industrial experience of around twenty six years. Before on experience, he has several publications in Internal and National renowned journals and conferences. He has received NDRC Invention award, IETE IRS award, DSIR National R\&D award at BEL, Best Counsellor award for IEEE-AIT student chapter. He is involved in providing research guidance to many M.Tech. and Ph.D. students and has successfully completed several corporate and industrial research projects. 